\title{
ANALYTICAL TREATMENT OF THE DELAYED FEEDBACK CONTROLLED LORENZ SYSTEM CLOSE TO A SUBCRITICAL HOPF BIFURCATION
}

\author{
V. Pyragas and K. Pyragas \\ Semiconductor Physics Institute, A. Goštauto 11, LT-01108 Vilnius, Lithuania \\ E-mail: viktpy@pfi.lt,pyragas@kes0.pfi.lt
}

Received 23 October 2007; revised 27 November 2007; accepted 22 February 2008

\begin{abstract}
We develop an analytical approach for the delayed feedback control of the Lorenz system close to a subcritical Hopf bifurcation. The periodic orbits arising at this bifurcation have no torsion and cannot be stabilized by a conventional delayed feedback control technique. We utilize a modification based on an unstable delayed feedback controller. The analytical approach employs the centre manifold theory, the near identity transformation, and averaging. We derive the characteristic equation for the Floquet exponents of the controlled orbit in an analytical form and obtain simple expressions for the threshold of stability as well as for an optimal value of the control gain. The analytical results are supported by numerical analysis of the original system of nonlinear differential-difference equations.
\end{abstract}

Keywords: chaos, dynamical systems, delayed feedback control, Lorenz system, subcritical Hopf bifurcation, centre manifold theory, near identity transformation, averaging

PACS: 05.45.Gg, 02.30.Yy, 02.30.Ks

\section{Introduction}

One of the most popular methods in chaos control research is the delayed feedback control (DFC) method [1]. The method allows a non-invasive stabilization of unstable periodic orbits (UPOs) of dynamical systems. To apply this method no exact knowledge of either the form of the periodic orbit or the system equations is needed. The delayed feedback control algorithm has been implemented successfully in experiments as diverse as electronic chaotic oscillators [2-5], mechanical pendulums [6,7], lasers [8-10], gas discharge systems [11-13], a current-driven ion acoustic instability [14], a chaotic Taylor-Couette flow [15], chemical systems [16,17], high-power ferromagnetic resonance [18], helicopter rotor blades [19], and a cardiac system [20]. In the literature, many interesting suggestions have been put forward for further application of the method (see Ref. [21] for review). Recently a challenging idea has been proposed [22-24] to use the DFC for controlling pathological brain rhythms.

The DFC method is based on the online measurement of a single output signal $s(t)$ that is a function of the current system state $x(t), s(t)=g[x(t)]$, and uses the time-delayed difference $s(t)-s(t-\tau)$ multiplied by a factor $K$ as a control signal. If delay time $\tau$ is equal to the period $T$ of an unstable periodic orbit of the system, the orbit may become stable under appropriate choice of the feedback strength $K$. The method is non-invasive in the sense that the control force $K[s(t)-s(t-\tau)]$ vanishes when the target state is reached.

Although the method is popular in experimental investigations, its theory is still in infancy. Systems with time delay are hard to handle because the dynamics take place in infinite-dimensional phase spaces. Even linear analysis of such systems is difficult due to infinite number of Floquet exponents characterizing the stability of controlled orbits. The linear and nonlinear analysis of such systems is usually performed numerically. So far, just one analytical result of a general character has been obtained. It has been proven that the method cannot stabilize UPOs with an odd number of real positive Floquet exponents (the odd number limitation) [25,26]. This is a topological limitation related to the absence of a torsion of the controlled UPO. In addition, there are some analytical results concerning quantitative estimation of the stability of UPOs subjected to delayed feedback [26,27], but they are of limited generality. Such estimations are elaborated only for UPOs arising from a flip bifurcation. 
In this context, a reasonable way for further development of the delayed feedback control theory is to look for problems allowing an analytical treatment. Our idea for analytical approach is to consider dynamical systems close to bifurcation points of periodic orbits. Some advances in this direction have been recently achieved for dynamical systems close to the subcritical Hopf [28] as well as Neimark-Sacker (discrete Hopf) [29] bifurcations.

In a short Letter [30], we have proposed a modified delayed feedback controller with an additional unstable mode in order to overcome the odd number limitation. The success of such a modification has been numerically demonstrated for the Lorenz system but no theoretical foundation has been presented. In this paper, we extend the ideas of Letter [30]. We develop a systematic analytical approach for delayed feedback control of dynamical systems close to a subcritical Hopf bifurcation. UPOs arising from this bifurcation have no torsion and cannot be stabilized by the conventional DFC technique. We demonstrate our approach for the Lorenz system as a representative of dynamical systems with torsion free unstable periodic orbits. Note, that the control of a simple second order dynamical system close to a subcritical Hopf bifurcation has been considered in Ref. [28]. However, the theory presented in Ref. [28] cannot be applied for high-dimensional systems. An analytical approach developed in this paper is applicable for any dynamical system with an arbitrary large phase space dimension.

The rest of the paper is organized as follows. In Sec. 2, we formulate the problem and introduce the control algorithm. Section 3 is devoted to the analysis of the free Lorenz system. By using the centre manifold theory, near identity transformation, and averaging, we obtain an analytical solution for an unstable limit cycle arising in the neighbourhood of the subcritical Hopf bifurcation. Then similar analysis is performed for the controlled Lorenz system in Sec. 4. In the end of that section, we analyse numerically the original system under delayed feedback control and confirm the validity of the analytical results. The paper is finished by conclusions presented in Sec. 5.

\section{Problem formulation}

We consider the paradigmatic chaotic system

$$
\begin{aligned}
& \dot{x}=\sigma(y-x), \\
& \dot{y}=r x-y-x z, \\
& \dot{z}=x y-b z,
\end{aligned}
$$

originally introduced by Lorenz [31] as a model of turbulent convection. In usual considerations of this system the parameters $\sigma$ and $b$ are fixed respectively to the values 10 and $8 / 3$, and analysis is performed for the variable parameter $r$. For $0<r<1$, the Lorenz system has a unique stable steady state (a stable node) at the origin $C^{0}:(0,0,0)$. For $r>1$, the origin becomes a saddle and two additional symmetrical stable fixed points $C^{ \pm}$, with coordinates

$$
\left(x_{f}^{ \pm}, y_{f}^{ \pm}, z_{f}\right)=( \pm \sqrt{b(r-1)}, \pm \sqrt{b(r-1)}, r-1),
$$

appear. For $r>r_{\mathrm{H}}$, the steady states $C^{ \pm}$become unstable. The value [32]

$$
r_{\mathrm{H}}=\frac{\sigma(\sigma+b+3)}{\sigma-b-1} \approx 24.7368
$$

represents the point at which the subcritical Hopf bifurcation occurs. Just below this bifurcation point, for

$$
r=r_{\mathrm{H}}-\Delta r, \quad 0<\Delta r \ll r_{\mathrm{H}},
$$

there are two small unstable limit cycles surrounding the stable steady states $C^{ \pm}$. Moreover, at the same values of the parameter $r$ there exists a strange attractor [32]. Thus the system is multistable and depending on initial conditions the phase trajectory may be either attracted to the one of the steady states or exhibit a chaotic behaviour on the strange attractor.

Our aim is to stabilize the unstable limit cycles arising at the Hopf bifurcation using the delayed feedback control technique. Especially we are interested in analytical treatment of this problem. Note that the periodic orbits arising at this bifurcation are torsion free and we need an unstable controller. Specifically, we consider the following control algorithm:

$$
\begin{aligned}
\dot{x} & =\sigma(y-x), \\
\dot{y} & =r x-y-x z+W\left(y-y_{f}\right), \\
\dot{z} & =x y-b z, \\
\dot{W} & =\Lambda_{c} W+K[y-y(t-\tau)] .
\end{aligned}
$$


Here as well as in Ref. [30] we suppose that $y$ is an observable and apply the control perturbation $W\left(y-y_{f}\right)$ only to the second equation of the Lorenz system. However, unlike the Ref. [30] we use a nonlinear perturbation. As pointed out in Ref. [28] this is a necessary requirement when considering the system close to a Hopf bifurcation in order to provide the coupling between the controlled system and controller in averaged equations. The parameter $y_{f}$ in the perturbation is the value of the observable when the system is in one of the stable steady states $C^{ \pm}$. For definiteness, we consider the control of the periodic orbit surrounding the fixed point $C^{+}$and take $y_{f} \equiv y_{f}^{+}=\sqrt{b(r-1)}$. Note that the value $y_{f}$ can be measured experimentally, since $C^{+}$is the stable fixed point.

Equation (5d) describes an unstable delayed feedback controller, which supplements the system with an additional unstable Floquet mode and eliminates the odd number limitation [30]. The positive parameter $\Lambda_{c}>0$ defines the value of the additional Floquet exponent. The parameter $K$ denotes the strength of the feedback gain. The delay time $\tau$ in Eq. (5d) is equal to the period $T$ of the unstable periodic orbit such that the controller does not change the periodic solutions of the Lorenz system with the period $T=\tau$. Thus if the stabilization of the periodic orbit is successful there is no power dissipated in the feedback loop.

In a real experiment, the period $T$ of an UPO is not known a priory and different strategies can be used for selection of the right value of the delay time $\tau$. A universal approach is based on minimization of the amplitude of the feedback perturbation $K[y-y(t-\tau)]$ [1]. An adaptive technique with automatic adjustment of the delay time has been considered in Ref. [33]. Another approach is based on minimization of the difference $\tau-T_{y}(\tau)$, where $T_{y}(\tau)$ is a period of the output signal $y(t)$, which generally differs from $T$ if $\tau \neq T$ [34].

\section{Analysis of the free Lorenz system}

We start our analysis with the free Lorenz system (1). First we transform the variables using the eigenvectors of the steady state $C^{+}$at the bifurcation point $r=r_{\mathrm{H}}$ as a basis for a new coordinate system. Then applying the centre manifold theory we eliminate a fast nonoscillating mode and obtain a reduced system for oscillating modes. Using the near identity transformation we transform the equations for the oscillating modes to the normal form of the subcritical Hopf bifurcation. As a final result of this section, we obtain an analytical so- lution for the unstable periodic orbit arising from this bifurcation.

\subsection{Transforming the system variables}

Our aim is the control of the unstable limit cycle surrounding the stable fixed point $C^{+}$. Thus it is convenient to shift the origin to this point by using the transformation

$$
x=x_{f}^{+}+u_{1}, \quad y=y_{f}^{+}+u_{2}, \quad z=z_{f}+u_{3} .
$$

Defining the state vector

$$
\boldsymbol{u}=\left(u_{1} u_{2} u_{3}\right)^{T}
$$

we rewrite the Lorenz equations in the matrix form

$$
\dot{\boldsymbol{u}}=A_{0} \boldsymbol{u}-\varepsilon A_{1} \boldsymbol{u}+\boldsymbol{N}(\boldsymbol{u}),
$$

where matrices $A_{0}, A_{1}, \boldsymbol{N}(\boldsymbol{u})$ are

$$
\begin{aligned}
& A_{0}=\left(\begin{array}{ccc}
-\sigma & \sigma & 0 \\
1 & -1 & -\sqrt{b\left(r_{\mathrm{H}}-1\right)} \\
\sqrt{b\left(r_{\mathrm{H}}-1\right)} & \sqrt{b\left(r_{\mathrm{H}}-1\right)} & -b
\end{array}\right), \\
& A_{1}=\left(\begin{array}{ccc}
0 & 0 & 0 \\
0 & 0 & -1 \\
1 & 1 & 0
\end{array}\right), \quad \boldsymbol{N}(\boldsymbol{u})=\left(\begin{array}{c}
0 \\
-u_{1} u_{3} \\
u_{1} u_{2}
\end{array}\right)
\end{aligned}
$$

and parameter

$\varepsilon=\sqrt{b\left(r_{\mathrm{H}}-1\right)}-\sqrt{b(r-1)} \approx \sqrt{b /\left(r_{\mathrm{H}}-1\right)} \Delta r / 2$

defines the closeness of the system to the bifurcation point $r=r_{\mathrm{H}}$. This is the main control parameter the smallness of which we exploit in the following perturbation theory.

The first two terms $A_{0} \mathbf{u}$ and $\varepsilon A_{1} \mathbf{u}$ on the right-hand side of Eq. (8) represent the linear part of the vector field, while the last term $\boldsymbol{N}(\boldsymbol{u})$ defines the nonlinear part. The matrix $A_{0}-\varepsilon A_{1}$ is the Jacobian derivative at the fixed point $C^{+}$, where $A_{0}$ is the value of the Jacobian calculated at the bifurcation point $r=r_{\mathrm{H}}$ and $\varepsilon A_{1}$ is a small deviation due to the shift of the parameter $r$ from the bifurcation point.

We now transform the system variables in such a way as to diagonalize the unperturbed linear part $A_{0} \boldsymbol{u}$ of the vector field. For this aim, we solve the eigenvalue problem for the matrix $A_{0}$,

$$
A_{0} \phi^{(\mu)}=\gamma_{\mu} \phi^{(\mu)} .
$$

As a result we obtain three eigenvalues

$$
\gamma_{1}=\gamma_{2}^{*} \equiv \mathrm{i} \omega \approx 9.6245 \mathrm{i}, \quad \gamma_{3} \approx-13.6667
$$


and three corresponding eigenvectors $\phi^{(1)}, \phi^{(2)}, \phi^{(3)}$. For compactness of presentation we do not write them out explicitly.

The first two eigenvalues are imaginary and their eigenvectors are complex conjugate. The third eigenvalue is real and negative. We use the eigenvectors $\phi^{(\mu)}$ as a basis for a new coordinate system and apply a linear transformation of the system variables:

$$
\boldsymbol{u}(t)=\sum_{\mu=1}^{3} \xi_{\mu}(t) \phi^{(\mu)} .
$$

The new dynamic variables $\xi_{\mu}(t)(\mu=1,2,3)$ define the amplitudes of the system eigenmodes at the bifurcation point $r=r_{\mathrm{H}}$. Note that $\xi_{1}(t)$ and $\xi_{2}(t)$ have to be complex conjugate, $\xi_{2}^{*}(t)=\xi_{1}(t)$, in order to provide the real-valued solution for $\boldsymbol{u}(t)$. To derive equations for the new variables $\xi_{\mu}(t)$ we insert Eq. (14) into system (8):

$$
\begin{aligned}
\sum_{\mu=1}^{3} \dot{\xi_{\mu}} \phi^{(\mu)}= & \sum_{\mu=1}^{3} \lambda_{\mu} \xi_{\mu} \phi^{(\mu)}-\varepsilon \sum_{\mu=1}^{3} A_{1} \phi^{(\mu)} \xi_{\mu} \\
& +N\left(\sum_{\mu=1}^{3} \xi_{\mu} \phi^{(\mu)}\right) .
\end{aligned}
$$

To obtain equations for $\xi_{\mu}(t)$ in the explicit form we have to solve the adjoint eigenvalue equation

$$
\boldsymbol{\psi}^{(\nu)} A_{0}=\gamma_{\nu} \boldsymbol{\psi}^{(\nu)},
$$

and obtain the left eigenvectors $\psi^{(\nu)}$ that satisfy the normalization conditions

$$
\left\langle\boldsymbol{\psi}^{(\nu)} \mid \boldsymbol{\phi}^{(\mu)}\right\rangle=\delta_{\nu \mu}, \quad \mu, \nu=1,2,3 .
$$

Multiplying Eq. (15) by $\psi^{(\nu)}$ from the left side and using conditions (17) one obtains finally the equations for the eigenmodes

$$
\begin{aligned}
\dot{\xi}_{\nu} & =\gamma_{\nu} \xi_{\nu}-\varepsilon \sum_{\mu=1}^{3}\left\langle\boldsymbol{\psi}^{(\nu)}\left|A_{1}\right| \boldsymbol{\phi}^{(\mu)}\right\rangle \xi_{\mu}+g_{\nu}\left(\xi_{1}, \xi_{2}, \xi_{3}\right) \\
& \equiv f_{\nu}\left(\xi_{1}, \xi_{2}, \xi_{3}\right), \quad \nu=1,2,3
\end{aligned}
$$

where $g_{\nu}\left(\xi_{1}, \xi_{2}, \xi_{3}\right)$ are the nonlinear functions

$$
g_{\nu}\left(\xi_{1}, \xi_{2}, \xi_{3}\right)=\left\langle\boldsymbol{\psi}^{(\nu)} \mid \boldsymbol{N}\left(\sum_{\mu=1}^{3} \xi_{\mu} \boldsymbol{\phi}^{(\mu)}\right)\right\rangle
$$

$\nu=1,2,3$.
Until now Eqs. (18) are exact. They are equivalent to the original Lorenz system (1). However, this form is more convenient when analysing the system dynamics close to the Hopf bifurcation, for small values of the parameter $\varepsilon$.

\subsection{Reducing the system dimension}

Close to the bifurcation point $\varepsilon=0$, Eqs. (18) admit an analytical treatment. First we note that for $\varepsilon=0$ the linear part of the vector field is diagonal and small deviations from the origin are described by three linear independent modes $\dot{\xi}_{\nu}=\gamma_{\nu} \xi_{\nu}, \nu=1,2,3$. The first two modes are oscillating, $\gamma_{1,2}= \pm \mathrm{i} \omega$, and the third mode is decaying, $\gamma_{3}<0$. This enables us to apply the centre manifold theory and exclude the decaying mode.

To get a reduced system of equations for the oscillating modes in relation to the parameter $\varepsilon$, we change for a time the role of the parameter $\varepsilon$. We regard $\varepsilon$ as an additional dependent variable that satisfies the trivial equation [32]

$$
\dot{\varepsilon}=0 \text {. }
$$

Then for the extended system (18), (20), linearized at the fixed point $\left(\xi_{1}, \xi_{2}, \xi_{3}, \varepsilon\right)=(0,0,0,0)$, the $\xi_{3}$ axis is a stable subspace and the $\left(\xi_{1}, \xi_{2}, \varepsilon\right)$ is the centre subspace. Thus according to the well-known theorem [32], in the $\left(\xi_{1}, \xi_{2}, \xi_{3}, \varepsilon\right)$ phase space there exists a centre manifold

$$
\xi_{3}=h\left(\xi_{1}, \xi_{2}, \varepsilon\right)
$$

tangent to the centre subspace

$$
\begin{aligned}
& h(0,0,0)=0 \\
& \left.\frac{\partial h}{\partial \xi_{1}}\right|_{(0,0,0)}=\left.\frac{\partial h}{\partial \xi_{2}}\right|_{(0,0,0)}=\left.\frac{\partial h}{\partial \varepsilon}\right|_{(0,0,0)}=0 .
\end{aligned}
$$

We expand the manifold function in Taylor series up to the second order terms:

$$
\begin{aligned}
h\left(\xi_{1}, \xi_{2}, \varepsilon\right)= & K_{200} \xi_{1}^{2}+K_{020} \xi_{2}^{2}+K_{002} \varepsilon^{2} \\
& +K_{011} \xi_{2} \varepsilon+K_{101} \xi_{1} \varepsilon+K_{110} \xi_{1} \xi_{2} .
\end{aligned}
$$

The linear terms are omitted to satisfy the conditions (22). Differentiating (21) we obtain

$$
\dot{\xi_{3}}=\frac{\partial h}{\partial \xi_{1}} \dot{\xi_{1}}+\frac{\partial h}{\partial \xi_{2}} \dot{\xi_{2}},
$$


or

$$
\begin{aligned}
& f_{3}\left(\xi_{1}, \xi_{2}, h\left(\xi_{1}, \xi_{2}, \varepsilon\right)\right)=\frac{\partial h}{\partial \xi_{1}} f_{1}\left(\xi_{1}, \xi_{2}, h\left(\xi_{1}, \xi_{2}, \varepsilon\right)\right) \\
& +\frac{\partial h}{\partial \xi_{2}} f_{2}\left(\xi_{1}, \xi_{2}, h\left(\xi_{1}, \xi_{2}, \varepsilon\right)\right) .
\end{aligned}
$$

By equating coefficients at different orders $\xi_{1}^{n} \xi_{2}^{m} \varepsilon^{l}(n+$ $m+l=2$ ) we get a linear system of equations yielding the coefficients of the expansion (23). Solving this system we obtain:

$$
\begin{aligned}
& K_{200} \approx-0.0047+0.0029 \mathrm{i}, \\
& K_{020}=K_{200}^{*}, \quad K_{002}=0, \\
& K_{011} \approx 0.0204-0.0025 \mathrm{i}, \\
& K_{101}=K_{011}^{*}, \quad K_{110} \approx 0.0041 .
\end{aligned}
$$

Substituting Eq. (21) in two first equations of the system (18) we obtain two equations for the oscillating modes:

$$
\dot{\xi_{\nu}}=f_{\nu}\left(\xi_{1}, \xi_{2}, h\left(\xi_{1}, \xi_{2}, \varepsilon\right)\right), \quad \nu=1,2 .
$$

Due to the property $\xi_{2}=\xi_{1}^{*}$ these equations are equivalent. Using the notations

$$
\xi_{1}=\xi, \quad \xi_{2}=\xi_{1}^{*}=\xi^{*}
$$

they can be presented in the form

$$
\dot{\xi}=f_{1}\left(\xi, \xi^{*}, h\left(\xi, \xi^{*}, \varepsilon\right)\right) .
$$

The function $f_{1}\left(\xi, \xi^{*}, h\left(\xi, \xi^{*}, \varepsilon\right)\right)$ in Eq. (29) is rather complicated. It contains terms up to the fourth order. However, most of them drop out when performing the averaging in order to transform this equation to a normal form. We write out explicitly only the relevant terms:

$$
\begin{aligned}
\dot{\xi}= & i \omega \xi+\varepsilon a_{10} \xi+a_{21}|\xi|^{2} \xi \\
& +a_{20} \xi^{2}+a_{02} \xi^{* 2}+a_{11}|\xi|^{2}+\ldots
\end{aligned}
$$

Here $a_{10}, a_{21}, a_{20}, a_{02}, a_{11}$ are the complex constants:

$$
\begin{aligned}
& a_{10} \approx-0.1803-1.0827 \mathrm{i}, \\
& a_{21} \approx 0.0005+0.0034 \mathrm{i}, \\
& a_{20} \approx 0.2218+0.2430 \mathrm{i}, \\
& a_{02} \approx 0.0124-0.0839 \mathrm{i}, \\
& a_{11} \approx-0.2804+0.2342 \mathrm{i} .
\end{aligned}
$$

Equation (30) represents the reduced system, which describes well the Lorenz dynamics close to the subcritical Hopf bifurcation. To obtain the solution for the unstable limit cycle arising from this bifurcation, in the next paragraph we transform this equation to the normal form.

\subsection{Near identity transformation and parameters of the unstable limit cycle}

The reduced system (30) can be transformed to the normal form of the subcritical Hopf bifurcation

$$
\dot{\eta}=\lambda_{f} \eta+c_{1} \eta^{2} \eta^{*}+O\left(|\eta|^{5}\right)
$$

either by using a perturbation theory based on the multiscaling expansion or by applying a near identity transformation. Both approaches lead to the same result, but the second approach is simpler to handle and we use it in this paper.

Following Ref. [32], the near identity transformation that transforms Eq. (30) to the form (32) is

$$
\xi=\eta+\sum_{2 \leq j+k \leq 3} \kappa_{j k} \frac{\eta^{j} \eta^{* k}}{j ! k !}+O\left(|\eta|^{4}\right)
$$

Substituting Eq. (33) in (30) and using Eq. (32) one obtains a polynomial equation with respect to $\eta$ and $\eta^{*}$. Equating coefficients of the polynomial equation yields the parameters $\lambda_{f}$ and $c_{1}$ of the normal form (32),

$$
\lambda_{f}=\mathrm{i} \omega+\varepsilon a_{10}
$$

$$
\begin{aligned}
c_{1} & =\frac{\mathrm{i}}{\omega}\left(a_{20} a_{11}-\frac{2}{3} a_{02} a_{02}^{*}-a_{11} a_{11}^{*}\right)+a_{21}+O(\varepsilon) \\
& \approx 0.0022-0.0233 \mathrm{i},
\end{aligned}
$$

and the coefficients $\kappa_{j k}$ of the near identity transformation (33). The coefficients of leading terms in the transformation are

$$
\kappa_{20}=\frac{2 a_{20}}{\lambda_{f}}, \kappa_{11}=\frac{a_{11}}{\lambda_{f}^{*}}, \kappa_{02}=\frac{a_{02}}{2 \lambda_{f}^{*}-\lambda_{f}}
$$

Equation (34) defines the eigenvalue of the fixed point $C^{+}$. For $\varepsilon>0$, it is stable since $\operatorname{Re}\left(\lambda_{f}\right)=$ $\varepsilon \operatorname{Re}\left(a_{10}\right)<0$. The unstable limit cycle surrounding this fixed point can be found by solving the normal form equation (32). By substitution $\eta=R \exp (\mathrm{i} \Theta)$, where 
$R$ and $\Theta$ are real-valued variables, this equation can be presented in the form

$$
\begin{aligned}
& \dot{\Theta}=\operatorname{Im}\left(\lambda_{f}\right)+\operatorname{Im}\left(c_{1}\right) R^{2}, \\
& \dot{R}=\left[\operatorname{Re}\left(\lambda_{f}\right)+\operatorname{Re}\left(c_{1}\right) R^{2}\right] R .
\end{aligned}
$$

From Eqs. (37b) and (34) it follows that the radius of the limit cycle is

$$
R_{0}=\sqrt{-\frac{\operatorname{Re}\left(a_{10}\right)}{\operatorname{Re}\left(c_{1}\right)}} \sqrt{\varepsilon} \approx 9.1472 \sqrt{\varepsilon} .
$$

The frequency of the limit cycle is determined by the right-hand side of Eq. (37a) at $R=R_{0}$ :

$$
\begin{aligned}
\omega_{0} & =\omega+\left[\operatorname{Im}\left(a_{10}\right)-\frac{\operatorname{Im}\left(c_{1}\right)}{\operatorname{Re}\left(c_{1}\right)} \operatorname{Re}\left(a_{10}\right)\right] \varepsilon \\
& \approx 9.6245-3.0345 \varepsilon .
\end{aligned}
$$

Thus the analytical solution of the normal form equation (32) corresponding to the unstable limit cycle is

$$
\eta_{0}(t)=R_{0} \exp \left(\mathrm{i} \omega_{0} t\right) .
$$

The dynamics of the mode $\xi$ corresponding to this solution are obtained by inserting Eq. (40) $\left(\eta=\eta_{0}(t)\right)$ in the transformation formula (33).

Finally, one can easily obtain the Floquet exponent $\Lambda_{0}$ of the unstable limit cycle. Linearization of Eq. (37b) at the fixed point $R=R_{0}$ leads to the expression

$$
\begin{aligned}
\Lambda_{0}= & \operatorname{Re}\left(\lambda_{f}\right)+3 \operatorname{Re}\left(c_{1}\right) R_{0}^{2}= \\
& -2 \operatorname{Re}\left(a_{10}\right) \varepsilon \approx 0.3607 \varepsilon .
\end{aligned}
$$

We see that the the Floquet exponent is proportional to the parameter $\varepsilon$ and is positive for $\varepsilon>0$.

\section{Analysis of the controlled Lorenz system}

In this section, we analyse the Lorenz system under delayed feedback control described by Eqs. (5). We exploit a relationship between the characteristic equations for the Floquet exponents of two different control problems, the delayed feedback control, and the proportional feedback control. The latter problem is much more simpler and admits an analytical treatment similar to that described in Sec. 3. As a result we obtain the characteristic equations for the Floquet exponents of the Lorenz system under delayed feedback control in analytical form. We finish the section with numerical analysis of the original system of nonlinear differentialdifference Eqs. (5).

\subsection{Proportional versus delayed feedback}

First, we rescale the controller variable and parameters

$$
W=\varepsilon w, \quad \Lambda_{c}=\varepsilon \lambda_{c}, \quad K=\varepsilon k
$$

to rewrite the controlled Lorenz system (5) in a more convenient form

$$
\begin{aligned}
& \dot{x}=\sigma(y-x), \\
& \dot{y}=r x-y-x z+\varepsilon w\left(y-y_{f}\right), \\
& \dot{z}=x y-b z, \\
& \dot{w}=\varepsilon \lambda_{c} w+k[y-y(t-\tau)] .
\end{aligned}
$$

Generally this is a rather complicated system of nonlinear differential-difference equations. The dynamics of the system take place in an infinite-dimensional phase space and reduction of the phase space dimension via the centre manifold theory is a nontrivial task. To overcome the problem of an infinite dimensional phase space we proceed in the following way.

Analogously to Ref. [27], we consider the proportional feedback control instead of the delayed feedback control, i.e., in Eq. (43d) we replace the delay term $y(t-\tau)$ with the periodic solution of the free Lorenz system $y_{0}(t)$ corresponding to the unstable limit cycle, which we intend to stabilize. Then in place of the system (43) we get

$$
\begin{aligned}
& \dot{x}=\sigma(y-x), \\
& \dot{y}=r x-y-x z+\varepsilon w\left(y-y_{f}\right), \\
& \dot{z}=x y-b z, \\
& \dot{w}=\varepsilon \lambda_{c} w+k\left[y-y_{0}(t)\right] .
\end{aligned}
$$

Both Eqs. (43) and (44) have the same periodic solution corresponding to the desired limit cycle. Although the limit cycle has different Floquet exponents for the systems (43) and (44), there exists a relationship between these two Floquet problems. 
In the case of the delayed feedback control, the Floquet exponents are determined by linearization of the system (43):

$$
\begin{aligned}
\delta \dot{x} & =\sigma(\delta y-\delta x), \\
\delta \dot{y} & =\left(r-z_{0}\right) \delta x-\delta y-x_{0} \delta z+\varepsilon\left(y_{0}-y_{f}\right) \delta w \\
\delta \dot{z} & =-b \delta z+x_{0} \delta y+y_{0} \delta x \\
\delta \dot{w} & =\varepsilon \lambda_{c} \delta w+k[\delta y-\delta y(t-\tau)] .
\end{aligned}
$$

Here $[\delta x, \delta y, \delta z]$ denote small deviations from the periodic orbit $\left[x_{0}(t), y_{0}(t), z_{0}(t)\right]=\left[x_{0}(t+\tau), y_{0}(t+\right.$ $\left.\tau), z_{0}(t+\tau)\right]$ that satisfies the free system (1), and $\delta w=w$. Due to the Floquet theory the delay term $\delta y(t-\tau)$ in Eq. (45d) can be eliminated and the system of differential-difference equations (45) can be transformed into the system of ordinary differential equations. The Floquet decomposition of solutions of the system (45) implies that $\delta y(t)=\exp (\Lambda t) U(t)$, where $\Lambda$ is the Floquet exponent and $U(t)=U(t-\tau)$ is a periodic function. It follows that the delay term can be expressed as $\delta y(t-\tau)=\exp (-\Lambda \tau) \delta y(t)$. The price one has to pay for the elimination of the delay term is that the variational equations (45) defining the Floquet exponent depend on the Floquet exponent itself.

In the case of the proportional feedback control the Floquet exponents are defined by linearization of the system (44). This leads to variational equations similar to Eqs. (45) with the only difference that the last term in Eq. (45d) $k[\delta y-\delta y(t-\tau)]=k[1-\exp (-\Lambda \tau)] \delta y$ is replaced by $k \delta y$. It follows that the Floquet exponents for the delayed feedback control can be obtained from linearized system (44) by using the substitution

$$
k \longrightarrow k[1-\exp (-\Lambda \tau)]
$$

Thus rather than analysing the system of differentialdifference Eqs. (43) we can focus on the analysis of more simple system (44) described by ordinary differential equations. If we manage to derive analytically a characteristic equation for the Floquet exponents of the system (44), then we can use the substitution (46) and obtain the characteristic equation for the case of the delayed feedback control system (43).

System (44) is nonautonomous due to the timedepended term $y_{0}(t)$ in Eq. (44d). It is convenient to transform this system to the autonomous form by supplementing it with an additional free Lorenz system

$$
\begin{aligned}
& \dot{x}_{0}=\sigma\left(y_{0}-x_{0}\right), \\
& \dot{y}_{0}=r x_{0}-y_{0}-x_{0} z_{0}, \\
& \dot{z}_{0}=x_{0} y_{0}-b z_{0} .
\end{aligned}
$$

We suppose that the initial conditions of the system (47) are chosen on the stable manifold of the desired limit cycle, such that its solution converges to the limit cycle. As a result these equations generate the periodic signal $y_{0}(t)=y_{0}(t+\tau)$ which is used as an input in Eq. (44d).

Our aim now is to reduce the dimension of the system (44), (47). But first we transform the variables in a similar way as it has been done in Sec. 3.1. We shift the origin in Eqs. (44), (47) to the fixed point $C^{+}$and obtain

$$
\begin{aligned}
\dot{\boldsymbol{u}} & =A_{0} \boldsymbol{u}-\varepsilon A_{1} \boldsymbol{u}+\left(\begin{array}{lll}
0 & 10
\end{array}\right)^{T} \varepsilon w u_{2}+\boldsymbol{N}(\boldsymbol{u}) \\
\dot{w} & =\varepsilon \lambda_{c} w+k\left[u_{2}-u_{02}\right] \\
\dot{\boldsymbol{u}}_{0} & =A_{0} \boldsymbol{u}_{0}-\varepsilon A_{1} \boldsymbol{u}_{0}+\boldsymbol{N}\left(\boldsymbol{u}_{0}\right)
\end{aligned}
$$

Here the vector $\boldsymbol{u}_{0}$ is defined analogously to the vector $\boldsymbol{u}$ in Eqs. (6) and (7),

$$
\begin{aligned}
\boldsymbol{u}_{0}= & \left(u_{01} u_{02} u_{03}\right)^{T}= \\
& \left(x_{0}-x_{f}^{+} y_{0}-y_{f}^{+} z_{0}-z_{f}\right)^{T} .
\end{aligned}
$$

Using the linear transformation (14) for the vector $\boldsymbol{u}$ and similar transformation

$$
\boldsymbol{u}_{0}(t)=\sum_{\mu=1}^{3} \xi_{0 \mu}(t) \phi^{(\mu)}
$$


for the vector $\boldsymbol{u}_{0}$ we finally transform the Lorenz system under proportional feedback control to the form

$$
\begin{aligned}
\dot{\xi}_{\nu}= & \gamma_{\nu} \xi_{\nu}-\varepsilon \sum_{\mu=1}^{3}\left\langle\boldsymbol{\psi}^{(\nu)}\left|A_{1}\right| \boldsymbol{\phi}^{(\mu)}\right\rangle \xi_{\mu} \\
& +\varepsilon w \sum_{\mu=1}^{3} \boldsymbol{\psi}_{2}^{(\nu)} \phi_{2}^{(\mu)} \xi_{\mu}+g_{\nu}\left(\xi_{1}, \xi_{2}, \xi_{3}\right) \equiv \\
& F_{\nu}\left(\xi_{1}, \xi_{2}, \xi_{3}, w\right), \quad \nu=1,2,3 \\
\dot{w}= & \varepsilon \lambda_{c} w+k \sum_{\mu=1}^{3} \phi_{2}^{(\mu)}\left[\xi_{\mu}-\xi_{0 \mu}\right] \\
\dot{\xi}_{0 \nu}= & \gamma_{\nu} \xi_{0 \nu}-\varepsilon \sum_{\mu=1}^{3}\left\langle\boldsymbol{\psi}^{(\nu)}\left|A_{1}\right| \phi^{(\mu)}\right\rangle \xi_{0 \mu} \\
& +g_{\nu}\left(\xi_{01}, \xi_{02}, \xi_{03}\right), \quad \nu=1,2,3
\end{aligned}
$$

convenient for application of the centre manifold theory. The nonlinear functions $g_{\nu}$ are defined by Eq. (19).

For small values of the parameters $\varepsilon, \lambda_{c}$, and $k$, the system (51) can be treated analytically in much the same way as the free Lorenz system.

\subsection{Reducing the dimension of the Lorenz system controlled by proportional feedback}

First we reduce the dimension of the system (51) using the technique described in Sec. 3.2. For $\varepsilon=0$, $\lambda_{c}=0$, and $k=0$, the linear part of the vector field is diagonal and small deviations from the origin are described by $\dot{\xi}_{\nu}=\gamma_{\nu} \xi_{\nu}, \dot{w}=0$, and $\dot{\xi}_{0 \nu}=\gamma_{\nu} \xi_{0 \nu}$ with $\nu=1,2,3$. The modes $\xi_{1}, \xi_{2}, \xi_{01}, \xi_{02}$ are oscillating with the eigenvalues $\gamma_{1,2}= \pm \mathrm{i} \omega$, the mode $w$ has zero eigenvalue, and the modes $\xi_{3}, \xi_{03}$ are decaying, since $\gamma_{3}<0$. Thus one can apply the centre manifold theory and exclude the decaying modes.

In order to get a reduced system of equations in relation to the parameters $\varepsilon, \lambda_{c}$, and $k$, we regard them as additional dependent variables satisfying the trivial equations

$$
\dot{\varepsilon}=0, \quad \dot{\lambda}_{c}=0, \quad \dot{k}=0 .
$$

The extended phase space of the system (51), (52) is defined by dynamical variables $\left(\xi_{1}, \xi_{2}, \xi_{3}, \varepsilon, \xi_{01}\right.$, $\left.\xi_{02}, \xi_{03}, w, k, \lambda_{c}\right)$. For this system, linearized at the origin, the plane $\left(\xi_{3}, \xi_{03}\right)$ is a stable subspace and the $\left(\xi_{1}, \xi_{2}, \xi_{01}, \xi_{02}, \varepsilon, w, k, \lambda_{c}\right)$ is the centre subspace. Thus in the extended phase space there exists a centre manifold

$$
\left(\begin{array}{c}
\xi_{3} \\
\xi_{03}
\end{array}\right)=\left(\begin{array}{c}
h_{c}\left(\xi_{1}, \xi_{2}, \varepsilon, \xi_{01}, \xi_{02}, w, k, \lambda_{c}\right) \\
h_{0}\left(\xi_{01}, \xi_{02}, \varepsilon\right)
\end{array}\right) \equiv \boldsymbol{H}
$$

tangent to the centre subspace at the origin:

$$
\boldsymbol{H}(\mathbf{0})=\mathbf{0}, \quad D \boldsymbol{H}(\mathbf{0})=\mathbf{0} .
$$

Here $D \boldsymbol{H}$ denotes the Jacobian of derivatives with respect to all variables $\left(\xi_{1}, \xi_{2}, \xi_{01}, \xi_{02}, \varepsilon, w, k, \lambda_{c}\right)$ of the centre subspace. First we note, that the function $h_{0}$ depends not on all variables of the centre subspace but only on the subset $\left(\xi_{01}, \xi_{02}, \varepsilon\right)$. This is due to the fact that Eq. (51c) is independent of Eqs. (51a) and (51b). Equation (51c) corresponds to the free Lorenz system and coincides with Eq. (18). It follows that the manifold function $h_{0}$ coincides with that of Eq. (23), i.e., $h_{0}=h\left(\xi_{01}, \xi_{02}, \varepsilon\right)$, and hence

$$
\xi_{03}=h\left(\xi_{01}, \xi_{02}, \varepsilon\right) .
$$

Substituting Eq. (53) in (51) one obtains the equation for the manifold function $\boldsymbol{H}$, similar to Eq. (25). Direct analysis of this equation shows that the expansion of the function $h_{c}$ up to the second order terms also leads to the expression (23), i. e., $h_{c}\left(\xi_{1}, \xi_{2}, \varepsilon, \xi_{01}, \xi_{02}, w, K, \lambda_{c}\right)=$ $h\left(\xi_{1}, \xi_{2}, \varepsilon\right)$, and hence

$$
\xi_{3}=h\left(\xi_{1}, \xi_{2}, \varepsilon\right) .
$$

It turns out that the controller does not change the manifold function of the controlled system. Substituting Eq. (56) in the first two Eqs. (51a) (with $\nu=1,2$ ) one obtains the equation for the oscillating mode of the controlled system

$$
\dot{\xi}=F_{1}\left(\xi, \xi^{*}, h\left(\xi, \xi^{*}, \varepsilon\right), w\right),
$$

where $\xi=\xi_{1}=\xi_{2}^{*}$. Writing out explicitly only the relevant terms of the function $F_{1}$ one finally obtains the reduced equation for the controlled system in the form

$$
\begin{gathered}
\dot{\xi}=\mathrm{i} \omega \xi+\varepsilon a_{10} \xi+\varepsilon b_{11} w \xi+a_{21}|\xi|^{2} \xi+ \\
+a_{20} \xi^{2}+a_{02} \xi^{* 2}+a_{11}|\xi|^{2}+\ldots
\end{gathered}
$$

similar to Eq. (30). The only difference is that here we have an additional term $\varepsilon b_{11} w \xi$ related to the control perturbation. The coefficients $a_{10}, a_{21}, a_{20}, a_{02}, a_{11}$ are defined by Eq. (31) and the value of the coefficient $b_{11}$ is

$$
b_{11} \approx 0.4278-0.0505 \mathrm{i} \text {. }
$$


Equation (58) has to be supplemented by Eq. (51b) for the variable $w$ to complete the system of reduced equations describing the dynamics of the Lorenz system under proportional feedback control. Writing explicitly the sum in Eq. (51b) and substituting Eqs. (55) and (56) for the decaying modes, one obtains

$$
\begin{aligned}
\dot{w}= & \varepsilon \lambda_{c} w+k \phi_{2}^{(1)}\left(\xi-\xi_{0}\right)+k \phi_{2}^{(2)}\left(\xi^{*}-\xi_{0}^{*}\right) \\
& +k \phi_{2}^{(3)}\left[h\left(\xi, \xi^{*}, \varepsilon\right)-h\left(\xi_{0}, \xi_{0}^{*}, \varepsilon\right)\right] .
\end{aligned}
$$

Here $\xi_{0}=\xi_{01}=\xi_{02}^{*}$ is the solution of the free Lorenz system for the oscillating mode corresponding to the unstable limit cycle.

\subsection{Near identity transformation and averaging}

We use the near identity transformation (33) to simplify the reduced system (58), (60). First we transform Eq. (58). By substituting Eq. (33) in Eq. (58) and using technique described in Sec. 3.3 we derive the normal form equation similar to Eq. (32) but with an additional control perturbation $\varepsilon b_{11} w \eta$ :

$$
\dot{\eta}=\lambda_{f} \eta+c_{1} \eta^{2} \eta^{*}+\varepsilon b_{11} w \eta .
$$

Here we have restricted ourselves with the leading term in the control perturbation, i.e., in the expression $\varepsilon b_{11} w \xi=\varepsilon b_{11} w\left(\eta+O\left(|\eta|^{2}\right)\right)$ we have omitted the $O\left(|\eta|^{2}\right)$ terms.

We now simplify Eq. (60). Again we use the near identity transformation (33) and transform the variables $\left(\xi, \xi_{0}\right) \longrightarrow\left(\eta, \eta_{0}\right)$. Afterwards we average Eq. (60) over the period $\tau=2 \pi / \omega_{0}$ of the limit cycle. We suppose that $w$ is a slowly varying variable. The variable $\eta_{0}$ is defined by Eq. (40), i. e., $\eta_{0}(t)=R_{0} \exp \left(\mathrm{i} \omega_{0} t\right)$. For the variable $\eta$, we suppose that it can be presented in the form $\eta(t)=A(\varepsilon t) \exp \left(\mathrm{i} \omega_{0} t\right)$, where $A(\varepsilon t)$ is a slowly varying complex amplitude. Then the averaging eliminates all the terms containing the fast exponents, $\exp \left( \pm \mathbf{i} m \omega_{0} t\right)$, with nonzero integer $m$, and we obtain

$$
\dot{w}=\varepsilon \lambda_{c} w+k P\left(|\eta|^{2}-\left|\eta_{0}\right|^{2}\right),
$$

where coefficient $P$ is

$$
\begin{aligned}
P & =\frac{\mathrm{i}}{\omega}\left(a_{11} \phi_{2}^{(1)}-a_{11}^{*} \phi_{2}^{(2)}\right)+K_{110} \phi_{2}^{(3)}+O(\varepsilon) \\
& \approx-0.0243 .
\end{aligned}
$$

In the next paragraph, we exploit the simplified system of Eqs. (61), (62) to obtain the characteristic equation for the Floquet exponents of the controlled system.

\subsection{Stability analysis of the controlled system}

By substitution $\eta=R \exp (\mathrm{i} \Theta)$, Eqs. (61), (62) can be presented in the form

$$
\begin{aligned}
& \dot{\Theta}=\operatorname{Im}\left(\lambda_{f}\right)+\operatorname{Im}\left(c_{1}\right) R^{2}+\varepsilon \operatorname{Im}\left(b_{11}\right) w, \\
& \dot{R}=\left[\operatorname{Re}\left(\lambda_{f}\right)+\operatorname{Re}\left(c_{1}\right) R^{2}+\varepsilon \operatorname{Re}\left(b_{11}\right) w\right] R, \\
& \dot{w}=\varepsilon \lambda_{c} w+k P\left(R^{2}-R_{0}^{2}\right) .
\end{aligned}
$$

These equations have a solution $(\Theta, R, w)=$ $\left(\omega_{0} t, R_{0}, 0\right)$, which corresponds to the limit cycle of the free system. Linearization about this solution leads to the variational equations

$$
\begin{aligned}
& \delta \dot{\Theta}=2 \operatorname{Im}\left(c_{1}\right) R_{0} \delta R+\varepsilon \operatorname{Im}\left(b_{11}\right) \delta w, \\
& \delta \dot{R}=\Lambda_{0} \delta R+\varepsilon \operatorname{Re}\left(b_{11}\right) R_{0} \delta w, \\
& \delta \dot{w}=2 k P R_{0} \delta R+\varepsilon \lambda_{c} \delta w .
\end{aligned}
$$

Here $\Lambda_{0}$ is the Floquet exponent of the free orbit defined by Eq. (41). Equations (65b), (65c) are independent of Eq. (65a) and define the non-zero Floquet exponents of the controlled limit cycle, while Eq. (65a) defines the zero Floquet exponent. The non-zero Floquet exponents $\Lambda$ satisfy the quadratic equation

$$
\Lambda^{2}-\left(\Lambda_{0}+\varepsilon \lambda_{c}\right) \Lambda+\varepsilon \Lambda_{0} \lambda_{c}+\varepsilon^{2} Q k=0,
$$

where

$$
Q=2 P \operatorname{Re}\left(b_{11}\right) \operatorname{Re}\left(a_{10}\right) / \operatorname{Re}\left(c_{1}\right) \approx 1.7432 .
$$

We recall that Eq. (66) is the characteristic equation for the Floquet exponents of the limit cycle under proportional feedback control. To derive the characteristic equation for the case of the delayed feedback control we apply the substitution (46). As a result we obtain the quasipolynomial characteristic equation

$$
\begin{aligned}
& \Lambda^{2}-\left(\Lambda_{0}+\varepsilon \lambda_{c}\right) \Lambda+\varepsilon \Lambda_{0} \lambda_{c} \\
& \quad+\varepsilon^{2} Q k[1-\exp (-\Lambda \tau)]=0 .
\end{aligned}
$$

By rescaling the Floquet exponents

$$
\Lambda=\varepsilon \lambda, \quad \Lambda_{0}=\varepsilon \lambda_{0},
$$

this equation can be presented in a more convenient form

$$
\begin{aligned}
& \lambda^{2}-\left(\lambda_{0}+\lambda_{c}\right) \lambda+\lambda_{0} \lambda_{c} \\
& \quad+Q k[1-\exp (-\varepsilon \lambda \tau)]=0 .
\end{aligned}
$$


From Eqs. (41) and (69) it follows that

$$
\lambda_{0} \approx 0.3607 \text {. }
$$

Equation (70) is the main result of this paper. It defines the Floquet exponents of the controlled Lorenz system close to the subcritical Hopf bifurcation in relation to the bifurcation parameter $\varepsilon$ and the parameters $\lambda_{c}$ and $k$ of the unstable delayed feedback controller.

The quasipolynomial Eq. (70) has an infinite number of solutions, since it defines the Floquet exponents of a system described by differential-difference equations. In the general case, the solutions of Eq. (70) can be determined numerically. However, the leading Floquet exponents close to the bifurcation point can be obtained analytically. For $\varepsilon|\lambda| \tau \ll 1$, we can use an approximation $\exp (-\varepsilon \lambda \tau) \approx 1-\varepsilon \lambda \tau$, which transforms Eq. (70) into the simple quadratic equation

$$
\lambda^{2}-\left(\lambda_{0}+\lambda_{c}-k Q \varepsilon \tau\right) \lambda+\lambda_{0} \lambda_{c}=0 .
$$

The solutions of this equation are

$$
\begin{aligned}
\lambda_{1,2}= & \frac{\lambda_{0}+\lambda_{c}-k Q \varepsilon \tau}{2} \\
& \pm \sqrt{\frac{\left(\lambda_{0}+\lambda_{c}-k Q \varepsilon \tau\right)^{2}}{4}-\lambda_{0} \lambda_{c}} .
\end{aligned}
$$

In Fig. 1(a), we compare the leading Floquet exponents of the controlled system determined by three different methods, namely, (i) by solving the quasipolynomial Eq. (70), (ii) using the solutions (73) of the quadratic equation (72), and (iii) by solving the exact system of variational Eqs. (45). Equation (70) has been solved by the Newton-Raphson algorithm. The numerical analysis of the variational Eqs. (45) has been performed by the algorithm described in Ref. [35]. All the three above results are in good quantitative agreement, as viewed in Fig. 1(a). Thus the leading Floquet exponents are reliably predicted by the simple analytical expression (73).

The mechanism of stabilization is evident from Fig. 1(b). For $k=0$, two real positive solutions of Eq. (72), $\lambda=\lambda_{0}$ and $\lambda=\lambda_{c}$, describe an unstable eigenvalue of the free system and the free controller, respectively. With increasing $k$, the eigenvalues approach each other on the real axis, then collide and pass to the complex plane. For $k=k_{0}$, where

$$
k_{0}=\left(\lambda_{0}+\lambda_{c}\right) / Q \varepsilon \tau,
$$

they cross the imaginary axis and move symmetrically into the left half-plane, i.e., both the system and the
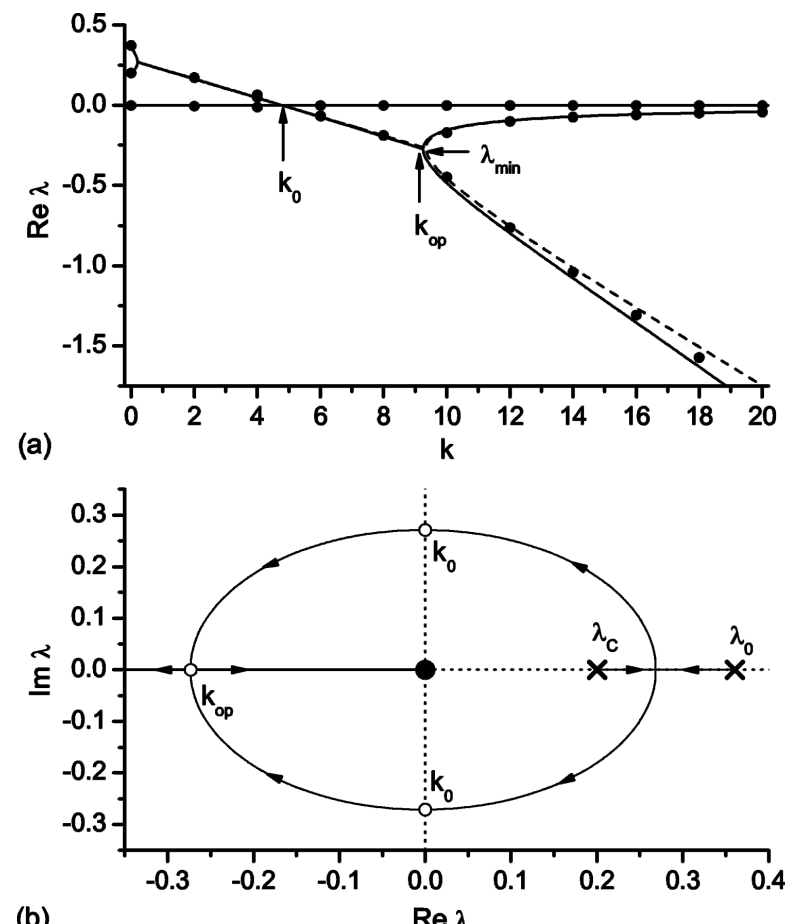

(b)

Fig. 1. (a) Real parts of leading Floquet exponents of the controlled limit cycle as functions of the control gain for $\varepsilon=0.1$, $\lambda_{c}=0.2$. Dashed and solid lines show the solutions of the characteristic Eqs. (72) and (70), respectively. Dots correspond to the values of Floquet exponents obtained from the exact variational Eqs. (45). (b) Root loci of Eq. (70) as $k$ varies from 0 to $\infty$ for the same parameter values as in (a). Crosses and black dot denote the location of the roots for $k=0$ and $k=\infty$, respectively.

controller become stable. An optimal value of the control gain is

$$
k_{o p}=k_{0}+2 \sqrt{\lambda_{0} \lambda_{c}} / Q \varepsilon \tau,
$$

since it provides the fastest convergence to the stabilized limit cycle with the characteristic rate $\lambda_{\min }=-\sqrt{\lambda_{0} \lambda_{c}}$.

\subsection{Numerical demonstrations}

To verify the validity of the linear theory we have numerically investigated the original system of nonlinear differential-difference Eqs. (43). For the set of parameters $\varepsilon=0.1(r \approx 24.1439), \lambda_{c}=0.2, k=9.25$, $\tau \approx 0.6740$, the results are presented in Fig. 2. In numerical simulations, the controller is switched on only when the system is close to the desired periodic orbit and switched off when it is far away from the orbit. Specifically, we proceed in the following way. For large values of the quantity $\left|y-y_{\tau}\right|>Y_{\max }=1.2$, we turn off the controller, i.e., we take $k=0$ and eliminate the term $\varepsilon w\left(y-y_{f}\right)$ in Eq. (43b). The controller variable is dropped to zero, $w=0$, at every moment of the turning off. 


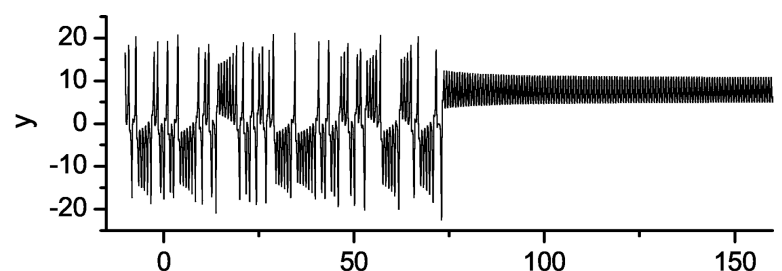

(a)

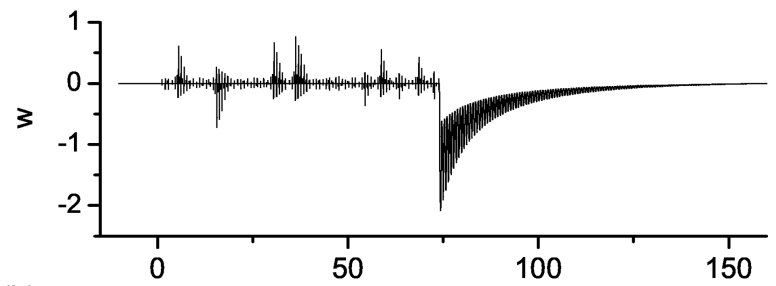

(b)

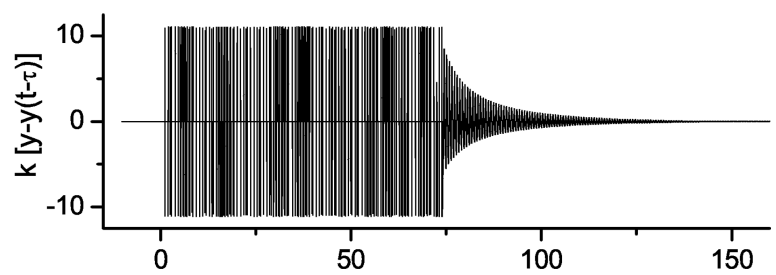

(c)

$\mathrm{t}$

Fig. 2. Dynamics of (a) variable $y$, (b) controller variable $w$, and (c) delayed feedback perturbation $k[y-y(t-\tau)]$. The initial conditions are $x(-15 \tau)=8.1096, y(-15 \tau)=13.0372, z(-15 \tau)=$ 14.2747, $w(-15 \tau)=0 . y(t)=0$ for $-15 \tau<t \leq-14 \tau$. The control is initiated at $t=\tau$. The values of the parameters are $\varepsilon=0.1, \lambda_{c}=0.2, \tau=0.6740, k=0$ for $-15 \tau \leq t<\tau$ and $k=9.25$ for $t \geq \tau$. For $|y-y(t-\tau)|>Y_{\max }=1.2$, the controller is off (see Sec. 4.5 for details). The black regions are densely filled by oscillations.

Without control $(t<\tau)$, the Lorenz system demonstrates a chaotic behaviour on the strange attractor. For $t>\tau$, the control algorithm starts to act and after a transient process the controlled system approaches a previously unstable limit cycle, and the feedback perturbation vanishes.

\section{Conclusions}

We have developed an analytical theory of the unstable delayed feedback controller proposed in Letter [30] for stabilization of unstable periodic orbits without torsion. The theory is applicable for any dynamical system close to a subcritical Hopf bifurcation. The periodic orbits arising at this bifurcation satisfy the odd number limitation and could not be stabilized by the conventional delay technique. Our analytical approach is demonstrated with the Lorenz system.

To compare our approach to that presented in Ref. [28] we note that a simple second order dynamical system under delayed feedback control considered in Ref. [28] has been treated analytically by the averaging method. Here, in the case of a more complex third order dynamical system, we had to utilize additional tools of nonlinear dynamics, namely, the centre manifold theory, near identity transformation, and the averaging. It should be recognized that instead of the near identity transformation the multi-scaling approach can be used. Both approaches lead to the same results, but we preferred the first approach in this paper. By using these tools we managed to derive analytically the characteristic equation for the Floquet exponents of the Lorenz system under delayed feedback control. Solving this equation we have determined simple analytical expressions for the leading Floquet exponents as well as for the threshold of stability and the optimal value of the control gain. Although the analytical approach has been demonstrated for the specific third order system, its extension to systems with the phase space dimension higher than three is straightforward.

We emphasize that the theory of the delayed feedback control is very complicated. Therefore any analytical results even though they are elaborated for a particular class of dynamic systems represent a valuable contribution to the theory of delayed feedback control. The analytical results obtained in this paper give a better insight into the mechanism of the delayed feedback control of unstable periodic orbits without torsion and are important for optimizing the control technique.

\section{References}

[1] K. Pyragas, Continuous control of chaos by selfcontrolling feedback, Phys. Lett. A 170, 421-427 (1992).

[2] K. Pyragas and A. Tamaševičius, Experimental control of chaos by delayed self-controlling feedback, Phys. Lett. A 180, 99-102 (1993).

[3] A. Kittel, J. Parisi, K. Pyragas, and R. Richter, Delayed feedback control of chaos in an electronic double-scroll oscillator, Z. Naturforsch. 49a, 843-846 (1994).

[4] D.J. Gauthier, D.W. Sukow, H.M. Concannon, and J.E.S. Socolar, Stabilizing unstable periodic orbits in a fast diode resonator using continuous time-delay autosynchronization, Phys. Rev. E 50, 2343-2346 (1994).

[5] P. Celka, Experimental verification of Pyragas's chaos control method applied to Chua's circuit, Int. J. Bifurcation Chaos Appl. Sci. Eng. 4, 1703-1706 (1994).

[6] T. Hikihara and T. Kawagoshi, Experimental study on stabilization of unstable periodic motion in magnetoelastic chaos, Phys. Lett. A 211, 29-36 (1996).

[7] D.J. Christini, V. In, M.L. Spano, W.L. Ditto, and J.J. Collins, Real-time experimental control of a sys- 
tem in its chaotic and nonchaotic regimes, Phys. Rev. E 56, R3749-R3752 (1997).

[8] S. Bielawski, D. Derozier, and P. Glorieux, Controlling unstable periodic orbits by a delayed continuous feedback, Phys. Rev. E 49, R971-R974 (1994).

[9] M. Basso, R. Genesio R, and A. Tesi, Controller design for extending periodic dynamics of a chaotic $\mathrm{CO}_{2}$ laser, Systems Control Lett. 31, 287-297 (1997).

[10] W. Lu, D. Yu, and R.G. Harrison, Instabilities and tracking of travelling wave patterns in a three-level laser, Int. J. Bifurcation Chaos Appl. Sci. Eng. 8, 17691775 (1998)

[11] T. Pierre, G. Bonhomme, and A. Atipo, Controlling a chaotic regime of nonlinear ionization waves using the time-delay autosynchronization method, Phys. Rev. Lett. 76, 2290-2293 (1996).

[12] E. Gravier, X. Caron, G. Bonhomme, T. Pierre, and J.L. Briançon, Dynamical study and control of drift waves in a magnetized laboratory plasma, Eur. J. Phys. D 8, 451-457 (2000).

[13] Th. Mausbach, Th. Klinger, A. Piel, A. Atipo, Th. Pierre, and G. Bonhomme, Continuous control of ionization wave chaos by spatially derived feedback signals, Phys. Lett. A 228, 373-380 (1997).

[14] T. Fukuyama, H. Shirahama, and Y. Kawai, Dynamical control of the chaotic state of the current-driven ion acoustic instability in a laboratory plasma using delayed feedback, Phys. Plasmas 9, 4525-4529 (2002).

[15] O. Lüthje, S. Wolff, and G. Pfister, Control of chaotic Taylor-Couette flow with time-delayed feedback, Phys. Rev. Lett. 86, 1745-1748 (2001).

[16] P. Parmananda, R Madrigal, M. Rivera, L. Nyikos, I.Z. Kiss, and V. Gaspar, Stabilization of unstable steady states and periodic orbits in an electrochemical system using delayed feedback control, Phys. Rev. E 59, 5266-5271 (1999).

[17] A. Guderian, A.F. Münster, M. Kraus, and F.W. Schneider, Electrochemical chaos control in a chemical reaction: Experiment and simulation, J. Phys. Chem. A 102, 5059-5064 (1998).

[18] H. Benner and W. Just, Control of chaos by timedelayed feedback in high-power ferromagnetic resonance experiments, J. Korean Phys. Soc. 40, 10461050 (2002).

[19] J.M. Krodkiewski and J.S. Faragher, Stabilization of motion of helicopter rotor blades using delayed feedback - modelling, computer simulation and experimental verification, J. Sound Vibration 234, 591-610 (2000).
[20] K. Hall, D.J. Christini, M. Tremblay, J.J. Collins, L. Glass, and J. Billette, Dynamic control of cardiac alternans, Phys. Rev. Lett. 78, 4518-4521 (1997).

[21] K. Pyragas, Delayed feedback control of chaos, Philos. Trans. R. Soc. London, Ser. A 364, 2309-2334 (2006).

[22] M.G. Rosenblum and A.S. Pikovsky, Controlling synchronization in an ensemble of globally coupled oscillators, Phys. Rev. Lett. 92, 114102 (2004).

[23] M.G. Rosenblum and A.S. Pikovsky, Delayed feedback control of collective synchrony: An approach to suppression of pathological brain rhythms, Phys. Rev. E 70, 041904 (2004).

[24] O.V. Popovych, C. Hauptmann, and P.A. Tass, Effective desynchronization by nonlinear delayed feedback, Phys. Rev. Lett. 94, 164102 (2005).

[25] H. Nakajima, On analytical properties of delayed feedback control of chaos, Phys. Lett. A 232, 207-210 (1997).

[26] W. Just, T. Bernard, M. Ostheimer, E. Reibold, and H. Benner, Mechanism of time-delayed feedback control, Phys. Rev. Lett. 78, 203-206 (1997).

[27] K. Pyragas, Analytical properties and optimization of time-delayed feedback control, Phys. Rev. E 66, 026207 (2002).

[28] K. Pyragas, V. Pyragas, and H. Benner, Delayed feedback control of dynamical systems at a subcritical Hopf bifurcation, Phys. Rev. E 70, 056222 (2004).

[29] T. Pyragienė and K. Pyragas, Delayed feedback control of forced self-sustained oscillations, Phys. Rev. E 72, 0260203 (2005).

[30] K. Pyragas, Control of chaos via an unstable delayed feedback controller, Phys. Rev. Lett. 86, 2265-2268 (2001).

[31] E.N. Lorenz, Deterministic non-periodic flow, J. Atmos. Sci. 20, 130-141 (1963).

[32] J. Guckenheimer and P. Holmes, Nonlinear Oscillations, Dynamical Systems, and Bifurcations of Vector Fields, Applied Mathematical Sciences Vol. 42 (Springer, New York, 1983).

[33] A. Kittel, J. Parisi, and K. Pyragas, Delayed feedback control of chaos by self-adapted delay time, Phys. Lett. A 198, 433-436 (1995).

[34] W. Just, D. Reckwerth, J. Mockel, E. Reibold, and H. Benner, Delayed feedback control of periodic orbits in autonomous systems, Phys. Rev. Lett. 81, 562-565 (1998).

[35] K. Pyragas, Control of chaos via extended delay feedback, Phys. Lett. A 206, 323-330 (1995).

\section{UŽDELSTO GRIŽTAMOJO RYŠIO VALDOMOS LORENCO SISTEMOS ANALIZINIS TYRIMAS SUBKRIZINĖS HOPFO BIFURKACIJOS APLINKOJE}




\section{Pyragas, K. Pyragas}

Puslaidininkiu fizikos institutas, Vilnius, Lietuva

\section{Santrauka}

Išplètojame analizinę teoriją uždelsto grižtamojo ryšio valdikliui (UGRV), valdančiam Lorenco sistemą, esančią arti subkrizinès Hopfo bifurkacijos taško. Šios bifurkacijos metu atsirandančiu periodinių orbitų topologija riboja UGRV, ir jų neįmanoma stabilizuoti iprastiniu UGRV metodu. Topologiniam ribojimui apeiti naudojame nestabilų valdiklį. Analiziniai tyrimai grindžiami centrinès daugdaros teorija, beveik tapačia transformacija bei vidurkinimo metodu. Analiziškai gauname charakteringas lygtis, kurių šaknys yra valdomos orbitos Flokè rodikliai. Taip pat gauname paprastas išraiškas, nusakančias valdiklio grižžtamojo ryšio stiprio stabilumo slenkstinę bei optimalią vertę. Analizinius rezultatus patvirtina išeitinès valdomos Lorenco sistemos skaitinis integravimas. 